\title{
Optimization of dose distributions of target volumes and organs at risk during stereotactic body radiation therapy for pancreatic cancer with dose-limiting auto- shells
}

Yangsen $\mathrm{CaO}^{\dagger}$, Xiaofei Zhu ${ }^{\dagger}$, Xiaoping Ju${ }^{\dagger}$, Yongming Liu, Chunshan Yu, Yongjian Sun, Zhitao Dai, Xueling Guo and Huojun Zhang*

\begin{abstract}
Background: To identify optimization of dose distributions of target volumes and decrease of radiation doses to normal tissues during stereotactic body radiation therapy (SBRT) for pancreatic cancer with dose-limiting auto-shells.

Methods: With the same prescription dose, dose constraints of normal organs and calculation algorithm, treatment plans of each eligible patient were re-generated with 3 shells, 5 shells and 7 shells, respectively. The prescription isodose line and beam number of each patient in 3-shell, 5-shell and 7-shell plan remained the same. Hence, a triplet data set of dosimetric parameters was generated and analyzed.
\end{abstract}

Results: As the increase of shell number, the conformal index, volumes encompassed by $100 \%$ prescription isodose line and 30\% prescription isodose line significantly decreased. The new conformal index was higher in 3-shell group than that in 5-shell and 7-shell group. A sharper dose fall-off was found in 5-shell and 7-shell group compared to 3shell group. And the tumor coverage in 7-shell was better than that of 3-shell and 5-shell. Lower $D_{5 c c}$ of the intestine, $D_{10 c c}$ of the stomach, $D_{\max }$ of the spinal cord and smaller $V_{10}$ of the spleen was confirmed in 7-shell group compared to 3-shell group.

Conclusions: More conformal dose distributions of target volumes and lower radiation doses to normal organs could be performed with the increase of dose-limiting auto-shells, which may be more beneficial to potential critical organs without established dose constraints.

Keywords: Auto-shells, Stereotactic body radiation therapy, Pancreatic cancer, Dose limiting

\section{Background}

High doses may be required to achieve local control attributable to inherent radio-resistance of pancreatic cancer. However, the abutting organs at risk (OAR), including the stomach, duodenum and bowel, limit the prescription dose because of radio-sensitivity. Due to the advances in the radiotherapy, the nonisocentric and noncoplanar technique of stereotactic body radiation

\footnotetext{
* Correspondence: chyyzhj@163.com

${ }^{\dagger}$ Equal contributors

Department of Radiation Oncology, Changhai Hospital affiliated to Navy

Medical University, 168 Changhai Road, Shanghai 200433, China
}

therapy (SBRT) could deliver precise radiation with high local dose conformation, precise target localization [1] with motion compensation strategies $[2,3]$ and facilitate quick dose fall-off outside the tumor volume $[4,5]$.

Nevertheless, treatment planning is strongly userdependent since optimization parameters were preselected [6, 7]. Hence, a well-balanced plan should be based on the ALARA (as-low-as-reasonably-achievable) principle, and is generally preferable with extreme quality in only a few categories as long as any strict critical structure limits are not violated. Owing to the normal tissues in close proximity to the pancreas, a plan with

(C) The Author(s). 2018 Open Access This article is distributed under the terms of the Creative Commons Attribution 4.0 International License (http://creativecommons.org/licenses/by/4.0/), which permits unrestricted use, distribution, and 
trade-offs, if clinical justified, among all planning objectives is required.

Traditionally, collimators are nontrivial for controlling dose conformity and dose fall-off. While multiple fixed collimators may allow better conformity and fewer hot spots around the tumor boundary than a single one, but it is time-consuming and a large number of collimators could even impair plan quality. The Iris collimator can reduce treatment time [8], but may not necessarily lead to better dosimetric plan quality [9]. Also, a larger tumor volume indicated a larger aperture collimator, contributing to a larger penumbra and slow dose fall-off, detrimental to normal organs.

Apart from the collimator, shell structures are also used for optimizing dose conformity and reducing skin entry doses as well as hot spots outside the target area. Furthermore, in addition to constraints of OARs, autoshell is required to control dose gradients around the target volume, but without too much compromise of target volume coverage. Therefore, the selection of the suited number of shell structures and distances between each shell is pivotal.

As a result, the aim of our study was to implement dose-limiting auto-shells in the SBRT for pancreatic cancer to minimize doses of OAR and non-critical organs and further optimize dose distributions of target volumes with Sequential Multi-Objective Optimization.

\section{Methods \\ Eligibility}

Medical records of patients with pancreatic cancer receiving SBRT in our center from October 2016 to April 2017 were reviewed. All patients received clinical examinations and abdominal CT or MRI scanning. The inclusion criteria were as follows: diagnose confirmed by pathological examinations; locally advanced pancreatic cancer; patients with resectable or borderline resectable cancer intolerant of surgical resections; age ranging from 18 to 75 years old; tumor located in the pancreatic head with maximum diameter $<5 \mathrm{~cm}$; ECOG $\leq 2$; all patients receiving the same prescription dose. The following exclusion criteria were used: patients with a history of radiotherapy prior to the SBRT; metastatic pancreatic cancer.

\section{Robotic radiosurgery system and treatment planning system}

SBRT was delivered via CyberKnife, an image-guided frameless stereotactic robotic radiosurgery system (Accuray Corporation, Sunnyvale CA). The treatment planning process was carried out with a dedicated treatment planning system, Multiplan version 4.0.2 (Accuray Inc.).
The sequential method was applied for all plans in the investigation. The inverse treatment-planning algorithm was performed to maximize the minimum dose to target volume or the mean dose, known as "optimize coverage (OCO)" in the system. The upper bounding constraints of the OAR were restricted to the following doses during the optimization (volume of interest limits, VOI limits): spinal cord: 3Gy; stomach: 15Gy; intestine: 14Gy; duodenum: $14 \mathrm{~Gy}$. In order to minimize the maximum doses to the critical structures, the above limits were stricter than those reported in the American Association of Physicists in Medicine guidelines in TG-101 [10]. The optimization of monitor unit (MU) was performed as follows: total MU: 90,000; max MU per beam: 500; max MU per node: 1500. This could reduce isodose lines showing up as streaks in the direction of beam entry points, and hot spots in the vicinity of the beam entry points just below the skin surface [11].

\section{Target delineations}

The procedure of CyberKnife was similar to our previous study $[12,13]$. Gross tumor volume (GTV) was delineated as a radiographically evident gross disease by contrast CT acquired from the portal-venous phase. At the discretion of the physician, clinical target volume (CTV) encompassing areas of the potential subclinical disease spread was also designated. In most cases, the CTV equaled GTV. A 2-5 mm expansion margin was included to determine the planning target volume (PTV). When the tumor was adjacent to critical organs, the expansion of PTV outside of CTV in this direction should be avoided. Therefore, the margin expansion was allowed to be nonuniform. References of normal tissue constraints were according to the American Association of Physicists in Medicine guidelines in TG-101 [10].

\section{Auto-shells planning}

Each patient treatment plan was re-generated with 3 shells, 5 shells and 7 shells, respectively. All the patients received the same prescription dose (37.5Gy/5f). At least $90 \%$ of PTV should be covered by the prescription dose. The prescription isodose line was limited to $70-80 \%$, which would restrict the tumor $\mathrm{D}_{\max }$. The prescription isodose line of each patient in 3-shell, 5-shell and 7-shell plan was the same. Shells were determined based on the expansions of PTV margins (Details shown in Table 1). The dose limitations between every two shells were assigned (Table 2) in order to obtain desired dose fall-off without compromising coverage significantly. The distance from the PTV margin to each shell was $5 \mathrm{~mm}$, $15 \mathrm{~mm}$ and $30 \mathrm{~mm}$ in 3-shell group, respectively; $2 \mathrm{~mm}$, $5 \mathrm{~mm}, 15 \mathrm{~mm}, 30 \mathrm{~mm}$ and $60 \mathrm{~mm}$ in 5-shell group, respectively; $2 \mathrm{~mm}, 5 \mathrm{~mm}, 10 \mathrm{~mm}, 15 \mathrm{~mm}, 30 \mathrm{~mm}$, $60 \mathrm{~mm}$ and $100 \mathrm{~mm}$ in 7-shell group, respectively. Beam 
Table 1 Generation of shells based on the expansions of PTV margins

\begin{tabular}{|c|c|c|c|c|c|c|c|}
\hline & \multicolumn{7}{|c|}{ Distance from each shell to the PTV margin $(\mathrm{mm})$} \\
\hline & 1st shell & 2nd shell & 3rd shell & 4th shell & 5th shell & 6th shell & 7th shell \\
\hline 3-shell group & 5 & 15 & 30 & - & - & - & - \\
\hline 5-shell group & 2 & 5 & 15 & 30 & 60 & - & - \\
\hline 7-shell group & 2 & 5 & 10 & 15 & 30 & 60 & 100 \\
\hline
\end{tabular}

reduction in the system was utilized to restrict beam numbers, remove low-weighted beams and reoptimize with the remaining beams to keep the plan quality, which resulted in ensuring the same number of beams in the three plans. The plans were calculated by Monte Carlo algorithm with high resolution and the uncertainty was $1 \%$.

\section{Statistical analysis}

Parameters selected for evaluation of doses of target volumes included conformity index (CI), new conformity index $(\mathrm{nCI})$, heterogeneity index (HI), gradient index (GI), coverage, volumes encompassed by $100 \%$ isodose line (100\% PD-V), volumes encompassed by $50 \%$ isodose line (50\% PD-V) and volumes encompassed by $30 \%$ isodose line (30\% PD-V). Crucially, the formula of calculation of CI was: $\mathrm{CI}=\frac{\text { prescription isodose volume }\left(P I V, \mathrm{~cm}^{3}\right)}{\text { tumor volume encompassed prescription isodose line }\left(T I V, \mathrm{~cm}^{3}\right)}$. The $\mathrm{nCI}$ was calculated as follows: $\mathrm{nCI}=\frac{C I}{\text { coverage }}$, in which coverage was defined as the ratio of target volumes covered with prescription dose to the target volume. The HI was determined by the following formula: $\mathrm{HI}=\frac{D_{\max }}{R_{x D D s e}}$, in which $\mathrm{R}_{\mathrm{xDose}}$ was the prescription dose. The definition of GI was the ratio of the volume of half the prescription isodose to the volume of the prescription isodose [14]. Evaluations of doses to OAR included the maximum dose $\left(D_{\max }\right)$ and volume doses. $D_{\max }$ was defined as the dose of a 0.035 -cc or less. The dose volume of the intestine, stomach and spinal cord was the dose of a 5-cc volume $\left(D_{5 c c}\right), 10-c c$ volume $\left(D_{10 c c}\right)$ and 0.35 -cc volume $\left(D_{0.35 c c}\right)$. Doses of a 5-cc and a 10-cc volume were investigated in the case of the duodenum. In addition to these OAR, doses to the spleen were also studied, including the mean dose $\left(D_{\text {mean }}\right)$, the dose of a half spleen volume $\left(\mathrm{D}_{50 \%}\right)$ and the volume receiving 5Gy $\left(\mathrm{V}_{5}\right)$ and 10Gy $\left(\mathrm{V}_{10}\right)$. However, 2 patients had splenectomy. Hence, dose evaluations were only performed in 18 patients.

Comparisons between parameters in different shell groups were based on analysis of variance (ANOVA) for a randomized block design. Fisher's least significance difference test (LSD) was performed when significant differences were found in the three groups after analysis of variance. Results were deemed significant at $P<0.05$. Statistical analyses were performed using IBM SPSS version 22.0 (SPSS Inc., Armonk, NY).

\section{Results}

\section{Patient characteristics}

A total of 20 patients were enrolled in the investigation. The median age was 64.5 years old. The prescription dose was $37.5 \mathrm{~Gy} / 5 \mathrm{f}\left(\mathrm{BED}_{10}=65.625 \mathrm{~Gy}\right.$, biological effective dose, $\alpha / \beta=10)$. The median GTV and PTV was $26.95 \mathrm{~cm}^{3}$ and $36.33 \mathrm{~cm}^{3}$, respectively. The beam number ranged from 150 to 210 and the median was 186 .

\section{Evaluations of dose distributions of PTV}

With the increase of shell number, the CI, 100\% PD-V and $30 \%$ PD-V significantly decreased (CI: 3 -shell vs 5 shell: $1.12 \pm 0.05$ vs $1.10 \pm 0.04, P<0.001$; 5 -shell vs 7 shell: $1.10 \pm 0.04$ vs $1.09 \pm 0.03, P=0.024$; 3 -shell vs 7 shell: $1.12 \pm 0.05$ vs $1.09 \pm 0.03, P<0.001)(100 \%$ PD-V: 3 -shell vs 5 -shell: $38.92 \pm 12.31$ vs $38.45 \pm 12.85, P=$ 0.036 ; 5 -shell vs 7 -shell: $38.45 \pm 12.85$ vs $37.73 \pm 12.62$, $P=0.002$; 3 -shell vs 7 -shell: $38.92 \pm 12.31$ vs $37.73 \pm$ 12.62, $P<0.001$ ) (30\% PD-V: 3 -shell vs 5 -shell: $344.53 \pm$ 119.93 vs $312.90 \pm 110.18, P<0.001$; 5 -shell vs 7 -shell: $312.90 \pm 110.18$ vs $299.15 \pm 106.41, P=0.006$; 3 -shell vs 7-shell: $344.53 \pm 119.93$ vs $299.15 \pm 106.41, P<0.001$ ).

Table 2 Dose limitations between every two shells

\begin{tabular}{|c|c|c|c|c|c|c|c|}
\hline & \multicolumn{7}{|c|}{ Dose limitations (Gy) } \\
\hline & $\begin{array}{l}\text { PTV margin to 1st } \\
\text { shell }\end{array}$ & $\begin{array}{l}\text { 1st shell to 2nd } \\
\text { shell }\end{array}$ & $\begin{array}{l}\text { 2nd shell to 3th } \\
\text { shell }\end{array}$ & $\begin{array}{l}\text { 3th shell to 4th } \\
\text { shell }\end{array}$ & $\begin{array}{l}\text { 4th shell to 5th } \\
\text { shell }\end{array}$ & $\begin{array}{l}\text { 5th shell to 6th } \\
\text { shell }\end{array}$ & $\begin{array}{l}\text { 6th shell to } 7 \text { th } \\
\text { shell }\end{array}$ \\
\hline $\begin{array}{l}\text { 3-shell } \\
\text { group }\end{array}$ & 26 & 17 & 9 & - & - & - & - \\
\hline $\begin{array}{l}\text { 5-shell } \\
\text { group }\end{array}$ & 32 & 26 & 17 & 9 & 6 & - & - \\
\hline $\begin{array}{l}\text { 7-shell } \\
\text { group }\end{array}$ & 32 & 26 & 21 & 17 & 9 & 6 & 5 \\
\hline
\end{tabular}


Additionally, there was significant difference between 3shell and 5-shell, 3-shell and 7-shell with respect to $\mathrm{nCI}$ and GI. Furthermore, the 50\% PD-V was smaller in 7shell compared with that of 3-shell and 5-shell. And the tumor coverage in 7-shell was better than that of 3-shell and 5-shell (Table 3).

\section{Evaluations of doses to OAR}

The doses to normal tissue were shown in Table 4. After restriction of doses to OAR with different shells, $D_{5 \mathrm{cc}}$ of the intestine, $D_{10 \mathrm{cc}}$ of the stomach and $D_{\max }$ of the spinal cord was lower in 7-shell than those in 3-shell $\left(\mathrm{D}_{5 \mathrm{cc}}\right.$ of the intestine: 3 -shell vs 7 -shell: $15.50 \pm 2.10$ vs $14.95 \pm 2.51, P=0.003 ; \mathrm{D}_{10 \mathrm{cc}}$ of the stomach: 3 -shell vs 7-shell: $14.32 \pm 1.74$ vs $13.97 \pm 2.23, P=0.020 ; \mathrm{D}_{\max }$ of the spinal cord: 3 -shell vs 7 -shell: $7.12 \pm 1.20$ vs $6.63 \pm$ $0.89, P=0.046)$, though only a trend of decrease of $\mathrm{D}_{10 \mathrm{cc}}$ of the stomach and $D_{\max }$ of the spinal cord was found when the number of shell increased.

Crucially, in addition to the conventional OARs, the dose-volume parameters of the spleen were further investigated. There was no significant difference between the three groups regarding $D_{\text {mean }}, V_{5}$ and $D_{50 \%}$. Nevertheless, $V_{10}$ was much smaller in 7 -shell group than that in 3-shell group.

Given that one patient with a large tumor abutting to the spleen, invading the splenic hilar, the radiation dose was much higher than that for other patients. Therefore, the further analysis of $V_{10}$ precluded the patient was performed. It was also shown that $V_{10}$ was smaller in 7shell group compared with 3-shell group (3-shell: $0 \pm$ 1.31; 5-shell: $0 \pm 1$.30; 7-shell: $0 \pm 0.82$; 3 -shell vs 5 -shell, $P=0.367$; 5 -shell vs 7 -shell: $P=0.156$; 3 -shell vs 7 -shell: $P=0.024)$. An exemplary image showing the comparison of dose contributions between the three shells, five shells and seven shells plan was illustrated (Fig. 1).

\section{Discussion}

Complex tumor shapes or lesions adjacent to critical organs were challenging to treatment plans, which however did not indicate multiple calibration of structures or complicated optimization steps [15]. Therefore, even a high-quality treatment plan, short and simple optimization scripts may be appropriate [14]. Besides, manual tuning parameters combined with iterative optimization with different scripts were based on tradeoffs between planning objectives, which were determined by clinical preferences and should be prioritized. Hence, a well-balanced plan required emphasis on several planning constraints and aims without any compromise of other vital goals. In our study, all of the treatment plans were generated with pre-selection of constraints, some of which were even stricter than those recommended, and optimization of PTV coverage (OCO), in order to meanwhile comply with ALARA. All other constraints remained unchangeable to explore the influence of shell structures on dose distributions.

The results of our study indicate that with the increase of the shells, CI, volumes encompassed by different isodose lines, doses to OAR with a certain volume and $V_{10}$ of the spleen decreased while dose conformity was better. In the Multiplan System, auto-shells are generated at the expansion of different diameters based on the PTV. Typically, the generation of auto-shells could be performed with a unique dilation value per direction, or with one value to all directions for symmetric dilations. Auto-shells are used to control dose distribution both close to and far away from a target. An auto-shell close to a target can modify conformity, while a shell distant to a target can be used to control low dose distributions or remote hot spots. Additionally, for those normal tissues not contoured as OAR, which were deemed possibly invulnerable to the radiation, hot spots could be avoided by auto-shells.

Table 3 Evaluations of dose distributions of PTV

\begin{tabular}{|c|c|c|c|c|c|c|c|}
\hline \multirow[b]{2}{*}{ Parameter } & \multicolumn{3}{|l|}{ Group } & \multirow{2}{*}{$\begin{array}{l}P \text {-value } \\
\text { (ANOVA) }\end{array}$} & \multirow{2}{*}{$\begin{array}{l}\text { P-value } \\
\text { (LSD, 3- } \\
\text { shell vs } \\
\text { 5-shell) }\end{array}$} & \multirow{2}{*}{$\begin{array}{l}P \text {-value } \\
\text { (LSD, 5- } \\
\text { shell vs } \\
\text { 7-shell) }\end{array}$} & \multirow{2}{*}{$\begin{array}{l}P \text {-value } \\
\text { (LSD, 3- } \\
\text { shell vs } \\
\text { 7-shell) }\end{array}$} \\
\hline & 3-shell & 5-shell & 7-shell & & & & \\
\hline $\mathrm{Cl}$ & $1.12 \pm 0.05$ & $1.10 \pm 0.04$ & $1.09 \pm 0.03$ & $<0.001$ & $<0.001$ & 0.024 & $<0.001$ \\
\hline $\mathrm{nCl}$ & $1.21 \pm 0.04$ & $1.19 \pm 0.03$ & $1.18 \pm 0.03$ & $<0.001$ & $<0.001$ & 0.185 & $<0.001$ \\
\hline $\mathrm{HI}$ & $1.44 \pm 0.01$ & $1.44 \pm 0.01$ & $1.44 \pm 0.01$ & 1.0 & 1.0 & 1.0 & 1.0 \\
\hline $\mathrm{Gl}$ & $3.03 \pm 0.14$ & $3.09 \pm 0.13$ & $3.07 \pm 0.14$ & 0.012 & 0.004 & 0.368 & 0.037 \\
\hline Coverage (\%) & $92.63 \pm 2.22$ & $92.60 \pm 1.83$ & $91.79 \pm 1.44$ & 0.016 & 0.904 & 0.014 & 0.010 \\
\hline 100\%_PD (cc) & $38.92 \pm 12.31$ & $38.45 \pm 12.85$ & $37.73 \pm 12.62$ & $<0.001$ & 0.036 & 0.002 & $<0.001$ \\
\hline $50 \% \_P D(c c)$ & $117.48 \pm 35.83$ & $117.65 \pm 36.53$ & $115.09 \pm 36.84$ & $<0.001$ & 0.749 & $<0.001$ & $<0.001$ \\
\hline $30 \% \_P D(c c)$ & $344.53 \pm 119.93$ & $312.90 \pm 110.18$ & $299.15 \pm 106.41$ & $<0.001$ & $<0.001$ & 0.006 & $<0.001$ \\
\hline MU & $54,674.51 \pm 13,149.78$ & $54,649.06 \pm 13,246.72$ & $54,058.67 \pm 12,744.23$ & 0.018 & 0.914 & 0.016 & 0.012 \\
\hline
\end{tabular}


Table 4 Evaluations of dose to OAR

\begin{tabular}{|c|c|c|c|c|c|c|c|}
\hline \multirow[b]{2}{*}{ Parameter } & \multicolumn{3}{|l|}{ Group } & \multirow{2}{*}{$\begin{array}{l}P \text {-value } \\
\text { (ANOVA) }\end{array}$} & \multirow[b]{2}{*}{$\begin{array}{l}P- \\
\text { value } \\
\text { (LSD, } \\
3- \\
\text { shell } \\
\text { vs 5- } \\
\text { shell) }\end{array}$} & \multirow[b]{2}{*}{$\begin{array}{l}P- \\
\text { value } \\
\text { (LSD, } \\
5- \\
\text { shell } \\
\text { vs 7- } \\
\text { shell) }\end{array}$} & \multirow[b]{2}{*}{$\begin{array}{l}P- \\
\text { value } \\
\text { (LSD, } \\
3- \\
\text { shell } \\
\text { vs 7- } \\
\text { shell) }\end{array}$} \\
\hline & 3-shell & 5-shell & 7-shell & & & & \\
\hline Intestine ( $\left.D_{\max }\right)$ & $21.69 \pm 3.13$ & $21.28 \pm 3.62$ & $21.17 \pm 3.64$ & 0.359 & 0.286 & 0.769 & 0.176 \\
\hline Intestine $\left(D_{5 c c}\right)$ & $15.50 \pm 2.10$ & $15.27 \pm 2.51$ & $14.95 \pm 2.51$ & 0.011 & 0.183 & 0.076 & 0.003 \\
\hline Stomach $\left(D_{\max }\right)$ & $22.14 \pm 2.12$ & $22.17 \pm 1.66$ & $22.12 \pm 2.35$ & 0.976 & 0.897 & 0.826 & 0.928 \\
\hline Stomach $\left(D_{10 c c}\right)$ & $14.32 \pm 1.74$ & $14.13 \pm 2.13$ & $13.97 \pm 2.23$ & 0.064 & 0.183 & 0.292 & 0.020 \\
\hline Duodenum $\left(\mathrm{D}_{\max }\right)$ & $18.23 \pm 4.04$ & $18.27 \pm 4.11$ & $17.91 \pm 4.01$ & 0.274 & 0.891 & 0.147 & 0.187 \\
\hline Duodenum $\left(\mathrm{D}_{5 \mathrm{5c}}\right)$ & $10.54 \pm 3.50$ & $10.72 \pm 3.55$ & $10.71 \pm 3.43$ & 0.63 & 0.395 & 0.961 & 0.422 \\
\hline Duodenum $\left(\mathrm{D}_{10 \mathrm{cc}}\right)$ & $8.76 \pm 3.22$ & $8.81 \pm 3.30$ & $8.80 \pm 3.20$ & 0.968 & 0.808 & 0.953 & 0.854 \\
\hline Spinal cord $\left(D_{\max }\right)$ & $7.12 \pm 1.20$ & $6.91 \pm 1.28$ & $6.63 \pm 0.89$ & 0.131 & 0.374 & 0.251 & 0.046 \\
\hline Spinal cord $\left(D_{0.35 c c}\right)$ & $6.56 \pm 1.05$ & $6.36 \pm 1.12$ & $6.17 \pm 0.75$ & 0.151 & 0.321 & 0.328 & 0.053 \\
\hline Spleen $\left(D_{\text {mean }}\right)$ & $31.99 \pm 11.91$ & $30.70 \pm 12.03$ & $30.34 \pm 11.38$ & 0.989 & 0.987 & 0.890 & 0.903 \\
\hline Spleen $\left(V_{5}\right)$ & $7.47 \pm 11.27$ & $5.22 \pm 11.88$ & $4.24 \pm 11.28$ & 0.851 & 0.697 & 0.873 & 0.584 \\
\hline Spleen $\left(V_{10}\right)$ & $0.005 \pm 5.42$ & $0 \pm 5.42$ & $0 \pm 5.23$ & 0.037 & 0.339 & 1.000 & 0.012 \\
\hline Spleen $\left(D_{50 \%}\right)$ & $26.60 \pm 5.73$ & $26.68 \pm 7.66$ & $26.96 \pm 5.31$ & 0.903 & 0.678 & 0.721 & 0.954 \\
\hline
\end{tabular}

In previous studies, it was suggested that a shell structure of 1-3 mm was used to limit the high dose conformity, while two auto-shells with a distance between each other of 5-10 $\mathrm{mm}$ for the optimization of the prescription dose conformity and $15-20 \mathrm{~mm}$ for the optimization of proximate dose fall-off. Additionally, a shell structure of $30-50 \mathrm{~mm}$ was employed to limit the skin doses and hot spots outside the proximate target region [9]. Therefore, the number and distance of shell structures should be adjusted according to the target volume, clinical justifications and planning goals. Furthermore, Schlaefer et al. [15] concluded that an additional shell structure with a distance of $5 \mathrm{~mm}$ to the PTV should be used to control dose gradient around the
PTV. In this study, the distances between each shell were similar to those reported.

Yoon et al. [16] has reported clinical practice of different shells for brain metastases. In their study, the original CyberKnife plans were produced using one to three dose-limiting auto-shells at prescription dose level and low dose levels of 10 to $30 \%$ of PD. The modified CyberKnife plans were generated by five dose-limiting shells at prescription dose level, intermediate dose level of $50 \%$ of prescription dose, and low dose levels with an optimal shell-dilation size based on their experience. It was shown that there was no difference in $\mathrm{CI}$ and tumor coverage between two plans, while the normal tissue volume receiving $50 \%$ of prescription dose was significantly
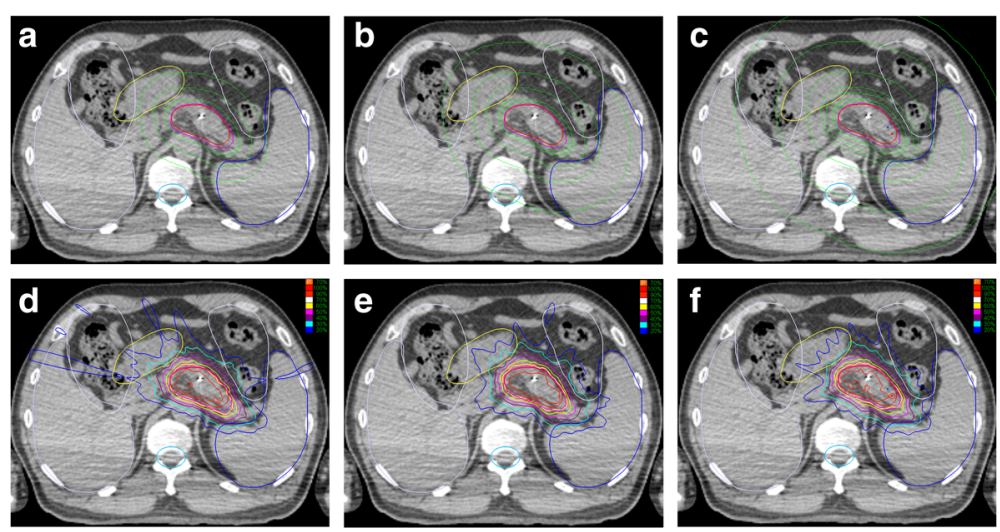

Fig. $\mathbf{1}$ a three-shell plan and $\mathbf{d}$ the dose distribution, $\mathbf{b}$ five-shell plan and $\mathbf{e}$ the dose distribution, $\mathbf{c}$ seven-shell plan and $\mathbf{f}$ the dose distribution 
decreased in the modified plans compared with the original plans by 1.28 -fold, indicating that a steeper dose fall-off could be achieved with the increase of autoshells.

The previous results were similar to ours. The GI was higher in 5-shell and 7-shell group compared with 3shell group. The underlying reason may be that the allowed distance for dose fall-off (37.5Gy to 26Gy) from the PTV margin to the first shell was $5 \mathrm{~mm}$ in 3-shell group. While in 5-shell and 7-shell group, the dose falloff (37.5Gy to 26Gy) was divided into two processes: the dose decreased from 37.5Gy to 32Gy within a short distance of $2 \mathrm{~mm}$ (from the PTV margin to the first shell), and from 32Gy to 26Gy within a distance of $3 \mathrm{~mm}$ (from the first shell to the second shell).

Additionally, it was elucidated that $\mathrm{V}_{10}$ of the spleen was significantly lower in 7-shell group than that in 3shell group, which may suggest that the radiation dose to the spleen was lower in 7-shell group.

\section{Conclusions}

Better conformity, lower radiation doses and smaller low-dose areas of normal organs could be achieved with the increase of dose-limiting auto-shells, though maybe at the cost of tumor coverage. Crucially, for organs without established dose constraints, milder radiationinduced toxicities may be attributable to auto-shells. Typically, protection of the spleen from excessive unintentional irradiation with auto-shells may reduce the incidence of lymphocytopenia, which may be beneficial to prognosis. Therefore, auto-shells could be an option to customize individual plans to ensure adequate normal organs sparing when clinically indicated.

\section{Abbreviations \\ 100\% PD-V: Volumes encompassed by 100\% isodose line; 30\% PD- V: Volumes encompassed by $30 \%$ isodose line; $50 \%$ PD-V: Volumes encompassed by $50 \%$ isodose line; ALARA: As-low-as-reasonably-achievable: ANOVA: Analysis of variance; Cl: Conformity index; CTV: Clinical target volume; Gl: Gradient index; GTV: Gross tumor volume; HI: Heterogeneity index; LSD: Fisher's least significance difference test; MU: Monitor unit; nCl: new conformity index; OAR: Organs at risk; OCO: Optimize coverage; PTV: Planning target volume; SBRT: Stereotactic body radiation therapy; VOI: Volumes of interest}

\section{Acknowledgements}

We appreciate Dr. Jiuhong Chen for her precise revisions and constructive comments.

\section{Funding}

This study was sponsored by China Health Promotion Foundation

(THC2015001) and Youth Fund of Changhai Hospital (CH201709).

Availability of data and materials

Not applicable.

\section{Authors' contributions}

CYS re-generated the plans. ZXF drafted the manuscript. JXP and ZHJ revised the manuscript. LYM, YCS and SYJ checked the plans. ZXF, DZT and GXL performed statistical analyses. ZHJ designed the study. All authors read and approved the final manuscript.
Ethics approval and consent to participate

The study was approved by the institutional review board of our hospital.

Consent for publication

The consents for publication of data have been obtained from patients.

Competing interests

The authors declare that they have no competing interests.

\section{Publisher's Note}

Springer Nature remains neutral with regard to jurisdictional claims in published maps and institutional affiliations.

Received: 11 September 2017 Accepted: 10 January 2018

Published online: 22 January 2018

\section{References}

1. Leksell $\mathrm{L}$. The stereotaxic method and radiosurgery of the brain. Acta Chir Scand. 1951;102:316-9.

2. Colombo F, Benedetti A, Pozza F, Zanardo A, Avanzo RC, Chierego G, et al. Stereotactic radiosurgery utilizing a linear accelerator. Appl Neurophysiol. 1985:48:133-45.

3. Hartmann GH, Schlegel W, Sturm V, Kober B, Pastyr O, Lorenz WJ. Cerebral radiation surgery using moving field irradiation at a linear accelerator facility. Int J Radiat Oncol Biol Phys. 1985;11:1185-92.

4. Dieterich S, Cleary K, D'Souza W, Murphy M, Wong KH, Keall P. Locating and targeting moving tumors with radiation beams. Med Phys. 2008;35:5684-94.

5. Chung HT, Kim DG. Modern radiosurgery equipment for treating brain metastases. Prog Neurol Surg. 2012;25:236-47.

6. Hoegele W, Loeschel R, Merkle N, Zygmanski P. An efficient inverse radiotherapy planning method for VMAT using quadratic programming optimization. Med Phys. 2012;39:444-54.

7. Ziegenhein P, Kamerling CP, Bangert M, Kunkel J, Oelfke U. Performanceoptimized clinical IMRT planning on modern CPUs. Phys Med Biol. 2013;58: 3705-15.

8. Echner GG, Kilby W, Lee M, Earnst E, Sayeh S, Schlaefer A, et al. The design, physical properties and clinical utility of an iris collimator for robotic radiosurgery. Phys Med Biol. 2009;54:5359-80.

9. Blanck O, Wang L, Baus W, Grimm J, Lacornerie T, Nilsson J, et al. Inverse treatment planning for spinal robotic radiosurgery: an international multiinstitutional benchmark trial. J Appl Clin Med Phys. 2016;17:313-30.

10. Benedict SH, Yenice KM, Followill D, Galvin JM, Hinson W, Kavanagh B, et al. Stereotactic body radiation therapy: the report of AAPM task group 101. Med Phys. 2010;37:4078-101.

11. Li T, Ozhasoglu C, Burton S, Flickinger J, Heron DE, Huq MS. A method to improve dose gradient for robotic radiosurgery. J Appl Clin Med Phys. 2015; 16:333-9.

12. Zhu $X$, Ju X, Cao F, Fang F, Qing S, Shen $Y$, et al. Safety and efficacy of stereotactic body radiation therapy combined with S-1 simultaneously followed by sequential S-1 as an initial treatment for locally advanced pancreatic cancer (SILAPANC) trial: study design and rationale of a phase II clinical trial. BMJ Open. 2016;6:e013220.

13. Zhu X, Li F, Ju X, Cao F, Cao Y, Fang F, et al. Prognostic role of stereotactic body radiation therapy for elderly patients with advanced and medically inoperable pancreatic cancer. Cancer Med. 2017; https://www.doi.org/10.1 002/cam4.1164.

14. Treuer H, Hoevels M, Luyken K, Visser-Vandewalle V, Wirths J, Kocher M, et al. Intracranial stereotactic radiosurgery with an adapted linear accelerator vs. robotic radiosurgery: comparison of dosimetric treatment plan quality. Strahlenther Onkol. 2015;191:470-6.

15. Schlaefer A, Schweikard A. Stepwise multi-criteria optimization for robotic radiosurgery. Med Phys. 2008;35:2094-103.

16. Yoon K, Cho B, Kwak J, Lee D, Kwon D, Ahn S, et al. Optimization of Cyberknife treatment planning using a dose-limiting auto-shells method for brain metastases. Med Phys. 2016;43:3371. 